\title{
Peter ADAMSON, A History of Philosphy Without Any Gaps, IV : Medieval Philosophy
}

Magali Roques

\section{(2) OpenEdition}

\section{Journals}

Édition électronique

URL : https://journals.openedition.org/ccm/7899

DOI : $10.4000 / \mathrm{ccm} .7899$

ISSN : 2119-1026

\section{Éditeur}

Centre d'études supérieures de civilisation médiévale/Université de Poitiers

\section{Édition imprimée}

Date de publication : 1 septembre 2021

Pagination : 242-244

ISBN : 978-2-490783-10-6

ISSN : 0007-9731

Référence électronique

Magali Roques, "Peter ADAmson, A History of Philosphy Without Any Gaps, IV : Medieval Philosophy ", Cahiers de civilisation médiévale [En ligne], 255 | 2021, mis en ligne le 01 septembre 2021, consulté le 10 février 2022. URL : http://journals.openedition.org/ccm/7899; DOI : https://doi.org/10.4000/ccm. 7899

\section{cc) (i) (2)}

La revue Cahiers de civilisation médiévale est mise à disposition selon les termes de la Licence Creative Commons Attribution - Pas d'Utilisation Commerciale - Pas de Modification 4.0 International. 
Peter Adamson, A History of Philosphy Without Any Gaps, IV : Medieval Philosophy, Oxford, Oxford University Press, 2019.

Dans cet ouvrage d'introduction à la philosophie médiévale, Peter Adamson, professeur de philosophie antique et arabe à l'Université de Munich, a pour ambition d'introduire à l'ensemble des productions philosophiques du monde latin médiéval, d'Alcuin à Pétrarque. Il s'agit du quatrième volume d'une « histoire sans omission » (« a history without any gaps »), les trois premiers ayant couvert la philosophie antique, la philosophie hellénistique et romaine, enfin la philosophie islamique. Cette somme à visée encyclopédique ne comprend pas moins de 78 chapitres de 6-8 p. chacun, agrémentés de $50 \mathrm{p}$. de notes ainsi que d'une bibliographie raisonnée qui mentionne les ouvrages de référence parus durant les trente dernières années ainsi que quelques ouvrages classiques plus anciens. Il se divise en trois parties d'égale longueur, la première portant sur les $\mathrm{XI}^{\mathrm{e}}-\mathrm{XII}^{\mathrm{e}} \mathrm{s}$., la deuxième sur le $\mathrm{XIII}^{\mathrm{e}} \mathrm{s}$. et la dernière sur le $\mathrm{XIV}^{\mathrm{e}} \mathrm{s}$. Avant toute chose, il faut souligner que l'ouvrage témoigne de qualités didactiques exceptionnelles. Les imposantes $540 \mathrm{p}$. peuvent se lire en continu, sans produire la moindre lassitude chez le lecteur - s'il est intéressé par la philosophie. En effet, le livre est d'abord à destination d'étudiants en philosophie. Pour cette seule raison, il mérite d'être recommandé à quiconque souhaite se familiariser avec les grandes figures de la philosophie et de la théologie médiévales. D'autres caractéristiques en font un ouvrage de référence. Elles proviennent de la volonté de l'a. de viser l'exhaustivité et de lutter contre la tendance, très forte dans le monde anglo-saxon, à réduire la philosophie médiévale à la seule figure de Thomas d'Aquin. Ce dernier a droit à trois chapitres, un quatrième visant à comparer son éthique à celle de son maître Albert le Grand. Il obtient ainsi le même traitement que Jean Duns Scot et Guillaume d'Ockham.

Le mot d'ordre de P. Adamson est que la philosophie médiévale ne prend sens que si elle est contextualisée, le contexte étant ici à comprendre au sens de « contexte historique et philosophique ». L'image qui se dégage de la philosophie médiévale latine est celle d'une « communauté conversationnelle», pour reprendre le concept forgé par la quatorziémiste Hester Goodenough Gelber (It Could Have Been Otherwise: Contingency and Necessity in Dominican Theology at Oxford: 1300-1350, Leyde/Boston, Brill [Studien und Texte zur Geistesgeschichte des Mittelalters, 81], 2004, p. 23-56). Le recours à l'idée de communauté conversationnelle est particulièrement approprié pour dépeindre avec vivacité la nature dialectique des textes philosophiques et théologiques du Moyen Âge latin. Cette idée produit également un effet de proximité avec le lecteur car elle permet d'insister sur ce qui rapproche les philosophes et théologiens médiévaux des autres philosophes du monde occidental depuis l'Antiquité, à savoir l'usage 
critique de la raison et la réflexion sur la valeur de cet usage.

Ce choix méthodologique n'est pas théorisé comme tel par l'a., sans doute pour ne pas briser le contrat tacite qui permet à l'exposé des doctrines d'être enjoué et plein d'humour. Comment décrire les présupposés méthodologiques implicites de P. Adamson? L'a. ne présuppose pas de distinction arbitraire entre philosophie et théologie comme cela a pu être pratiqué par les tenants de l'approche dite analytique de la philosophie médiévale, en vue d'insister sur l'actualité de la pensée des auteurs du Moyen Âge (voir par exemple les travaux de Claude Panaccio sur Guillaume d'Ockham). Il ne s'inspire pas non plus de la " philosophie chrétienne » théorisée par Étienne Gilson, une approche qui stipule que tout ce qui suit Thomas d'Aquin est de l'ordre de la pensée décadente. Au contraire, P. Adamson estime, à la suite de nombre de ses collègues formés à l'Université de Notre-Dame, que la philosophie du $\mathrm{XIV}^{\mathrm{e}} \mathrm{s}$. marque l'entrée dans la modernité, avec l'émergence de figures laïques qui écrivent hors de tout cadre universitaire, comme Marguerite Porète, Dante, Chaucer ou Julienne de Norwich, ainsi que l'émergence des productions philosophiques en langue vernaculaire. Sur le plan théorique, la modernité du XIV ${ }^{\mathrm{e}}$ s. se manifeste dans de nouvelles théories de la prédestination et de la grâce, qui seront discutées par Luther, ainsi que dans une nouvelle approche de la nature, avec une première tentative de mathématisation de la physique. La modernité du XIV ${ }^{\mathrm{e}} \mathrm{s}$. est également politique, puisque ce siècle voit la création des premières théories sécularistes du pouvoir politique.

Cette insistance sur la valeur philosophique et historique du $\mathrm{XIV}^{\mathrm{e}} \mathrm{s}$. n'a rien que de très convenu si l'on se réfère aux études médiévistiques des trente dernières années en Europe. Elle l'est moins dans les centres d'études médiévales du monde anglo-saxon. P. Adamson a le mérite d'entrer en dialogue avec les littéraires et les historiens, en ouvrant son corpus vers la littérature mystique et parodique et l'économie. C'est ainsi qu'un chapitre, d'ailleurs fort didactique, est consacré à l'économie médiévale. L'a. y présente les questions soulevées par la nature de la monnaie, la question du juste prix et la condamnation de l'usure (chap. 67). Les grandes productions littéraires revêtant un enjeu philosophique reçoivent également un traitement de faveur. On citera le Roman de la rose (chap. 45), les divers écrits de Dante (chap. 54), ainsi que Chaucer et Langland (chap. 72). On pourra d'ailleurs regretter que Boccace ne figure pas sur cette liste.

Plutôt que de multiplier les allusions aux poètes épris des questions d'éthique et de politique, P. Adamson a préféré insister sur un thème qui fait l'objet d'une attention de plus en plus soutenue de la part des médiévistes : la place des femmes dans la production du savoir au Moyen Âge. Un chapitre entier est consacré à la sexualité au Moyen Âge (chap. 73). En outre, dans ses présentations d'Hildegard von Bingen (chap. 19), de Hadewijch et Mechthild de Magdeburg (chap. 32), de Marguerite Porète (chap. 53) et de Catherine de Sienne (chap. 74), P. Adamson insiste sur l'idée que les femmes ont développé une forme spécifique de mysticisme, le mysticisme dit «affectif», pour reprendre la terminologie que P. Adamson emprunte à Christina van Dyke. Le mysticisme affectif s'oppose au mysticisme plus spéculatif que l'on peut retrouver chez les hommes, comme Maître Eckhart. Ce mysticisme féminin reposerait sur le lien spécifique établi, au Moyen Âge, entre la féminité et le corps, une thèse que $\mathrm{P}$. Adamson emprunte, non sans critiques ni nuances, à Carolyn Walker Bynum.

Le lecteur philosophe pourra se demander pourquoi une telle importance est accordée aux productions littéraires des femmes, alors que le droit médiéval ou la médecine sont passés sous silence. Leur valeur philosophique a été reconnue depuis longtemps et l'un comme l'autre ont fait l'objet de nombreux travaux de recherche en Europe. En effet, la médecine médiévale est riche d'explications sur le corps de la femme et elle aurait naturellement trouvé sa place aux côtés de la littérature. De même, le droit médiéval aurait pu être intégré aux développements sur la philosophie morale et politique des XIII et $\mathrm{XIV}^{\mathrm{e}} \mathrm{s}$.

L'accent mis sur la question des rapports entre littérature et morale témoigne de l'esprit d'une époque et marque la fin de l'approche analytique de la philosophie médiévale, qui privilégiait la philosophie de la logique et du langage. Comme dans les divers Companions et History of Medieval Philosophy parus les vingt dernières années chez Oxford University Press, Routledge, ou Brill - le plus souvent des œuvres collectives de grande qualité -, l'accent est davantage mis sur la métaphysique et la morale que sur les problématiques logiques qui ont été privilégiées dans les années 1960. Dans l'ouvrage de P. Adamson, il l'est d'ailleurs un peu 
trop. Les chapitres axés sur la logique médiévale (le chap. 22 sur la logique terministe, le chap. 58 sur la sémantique de Guillaume d'Ockham, le chap. 62 sur la logique du XIV ${ }^{\mathrm{e}} \mathrm{s}$.) sont un peu légers en comparaison avec le traitement de faveur accordé à la philosophie politique médiévale. Le manque le plus patent de l'ouvrage, d'ailleurs, concerne les écoles de logique du XII ${ }^{\mathrm{e}} \mathrm{s}$. Aucune mention n'est faite des Montani, Parvipontani, Melidunenses, Porretani, en dépit de leurs apports fondamentaux à l'histoire de la philosophie de la logique et du langage.

De même, la tradition de la logica moderna, qui se poursuivra jusqu'à l'âge classique, est fort peu représentée dans l'ouvrage. Un chapitre entier aurait pu être consacré à la logique des Calculateurs d'Oxford et à sa continuation jusqu'à la fin du Moyen Âge. On ne sera donc pas surpris par l'absence de toute référence au livre classique de Curtis Wilson sur la logique et la physique de William Heytesbury, un Calculateur anglais contemporain de Bradwardine et de Swineshead, auteur de Regulae solvendi sophismata dont la fortune sur le continent, aux XIV et $\mathrm{XV}^{\mathrm{e}} \mathrm{s}$., sera immense (Curtis WILson, William Heytesbury: Medieval Logic and the Rise of Mathematical Physics, Madison, University of Wisconsin Press [The University of Wisconsin Publications in Medieval Science, 3], 1956).

Il ne faudrait pas en conclure que la bibliographie en fin de volume est incomplète et partiale. Bien au contraire, la quantité des références et la sélection judicieuse à laquelle $\mathrm{P}$. Adamson a procédé sont tout à son honneur et font de cet ouvrage un outil de travail extrêmement utile aux étudiants comme aux chercheurs plus avancés. Les publications en anglais des maisons d'édition d'Oxford et de Cambridge sont certes au premier plan. Cependant, les publications les plus importantes des trente dernières années dans les trois grandes langues européennes (allemand, italien et français) y figurent en bonne et due place. Concernant la littérature francophone, on pourra mentionner les noms de Ruedi Imbach pour ses travaux sur la philosophie médiévale en langue vernaculaire, Alain de Libera pour ses travaux sur la mystique rhénane, Jean Jolivet pour ses travaux sur Abélard, Aurélien Robert (on notera l'erreur typographique sur le nom, p. 621) et Christophe Grellard pour leurs travaux sur l'atomisme, ou bien Joël Biard pour son étude sur Buridan. Les études allemandes sont relativement moins représentées que les études francophones. Si les travaux de Jürgen Miethke figurent en bonne place lorsqu'il est question des rapports entre l'Église et l'État, tout comme ceux de Dominik Perler lorsqu'il est question de scepticisme, l'école de Jan Aersten n'est pas mentionnée lorsqu'il est question de métaphysique.

Cette dernière omission n'est pas fortuite. P. Adamson n'écrit pas l'histoire des concepts ni celle des écoles de pensée. Il présente une conversation philosophique entre grands et moins grands auteurs, dans le but de montrer que la philosophie médiévale ne se limite pas à Thomas d'Aquin. Pour être compris à sa juste valeur, le docteur de l'Église doit être replacé « dans son contexte historique et intellectuel » (p. 281). Ce faisant, P. Adamson propose un livre solide, qui présente l'état actuel des savoirs en philosophie médiévale dans le monde anglo-saxon, et qui se distingue par la qualité du travail de documentation sur lequel il repose ainsi que par les qualités d'écriture dont il fait preuve.

Magali RoQues

UMR 7219 - SPHERE, Université de Paris 